\section{Platelet noradrenaline overload - cause of increased noradrenaline spillover in hypertension?}

\section{Shi-Sheng Zhou and Yiming Zhou}

We thank Barber-Chamoux and Esler for their Reply (Platelet noradrenaline uptake is unrelated to renal denervation. Nat. Rev. Cardiol. doi:10.1038/nrcardio.2016.144) ${ }^{1}$ to our Correspondence (Renal denervation for resistant hypertension: the wrong target? Nat. Rev. Cardiol. 13, 388; 2016) ${ }^{2}$, in which we argued why the current theoretical basis for renal denervation therapy - as described in the Perspectives article by Gulati et al. (Opinion: The rise, fall, and possible resurrection of renal denervation. Nat. Rev. Cardiol. 13, 238-244; 2016) ${ }^{3}-$ might be flawed. Barber-Chamoux and Esler raised several issues regarding our hypothesis that platelets are responsible for increased noradrenaline spillover in hypertension ${ }^{1}$. Before responding to these arguments, we would like briefly to outline the process of noradrenaline metabolism.

Noradrenaline metabolism can be divided into three stages: neuronal release-reuptake (upstream), blood cell-mediated clearance (midstream), and organ-mediated degradation and excretion (downstream) ${ }^{4}$ (FIG. 1). Indeed, increasing sympathetic nerve firing increases noradrenaline spillover. In the midstream stage, noradrenaline is degraded or inactivated by catechol-O-methyltransferase, phenolsulfotransferase, and monoamine oxidase in blood cells, and simultaneously concentrated and stored in platelets. Platelets serve as 'containers' to transport noradrenaline to downstream detoxifying/excretory organs for degradation and excretion. Each of the organs involved contributes to the total noradrenaline clearance rate (for example, renal insufficiency decreases noradrenaline clearance $)^{4}$. Clearly, midstream and downstream noradrenaline metabolism, although often neglected or underestimated, is also a determinant of noradrenaline spillover.

The role of neuronal uptake emphasized by Barber-Chamoux and Esler ${ }^{1}$ is highly questionable, because evidence for neuronal uptake of plasma noradrenaline came solely from pharmacological studies using noradrenaline-uptake inhibitors, especially desipramine (desmethylimipramine) ${ }^{5}$, which are known also to inhibit platelet uptake. Inhibition of platelet uptake has two effects: increasing plasma noradrenaline level and decreasing platelet noradrenaline release during platelet destruction. These effects might lead to regional differences in noradrenaline spillover in response to desipramine, owing to differences in platelet destruction between organs, with the heart having the lowest explain why intravenous desipramine infusion increases cardiac noradrenaline spillover, but decreases renal noradrenaline spillover ${ }^{7}$.

With regard to platelet noradrenaline uptake, in vitro evidence shows that platelets rapidly take up noradrenaline and convert it to a sulfated form ${ }^{8}$. Platelet noradrenalinedegrading enzymes help to maintain platelet noradrenaline at a stable level unless they are saturated by increased plasma noradrenaline (for example, in pheochromocytoma and hypertension). The finding that hypertension onset occurs following an increase in mean platelet volume ${ }^{4}$ suggests the importance of platelet-destruction rate ${ }^{6}$. This discrepancy can

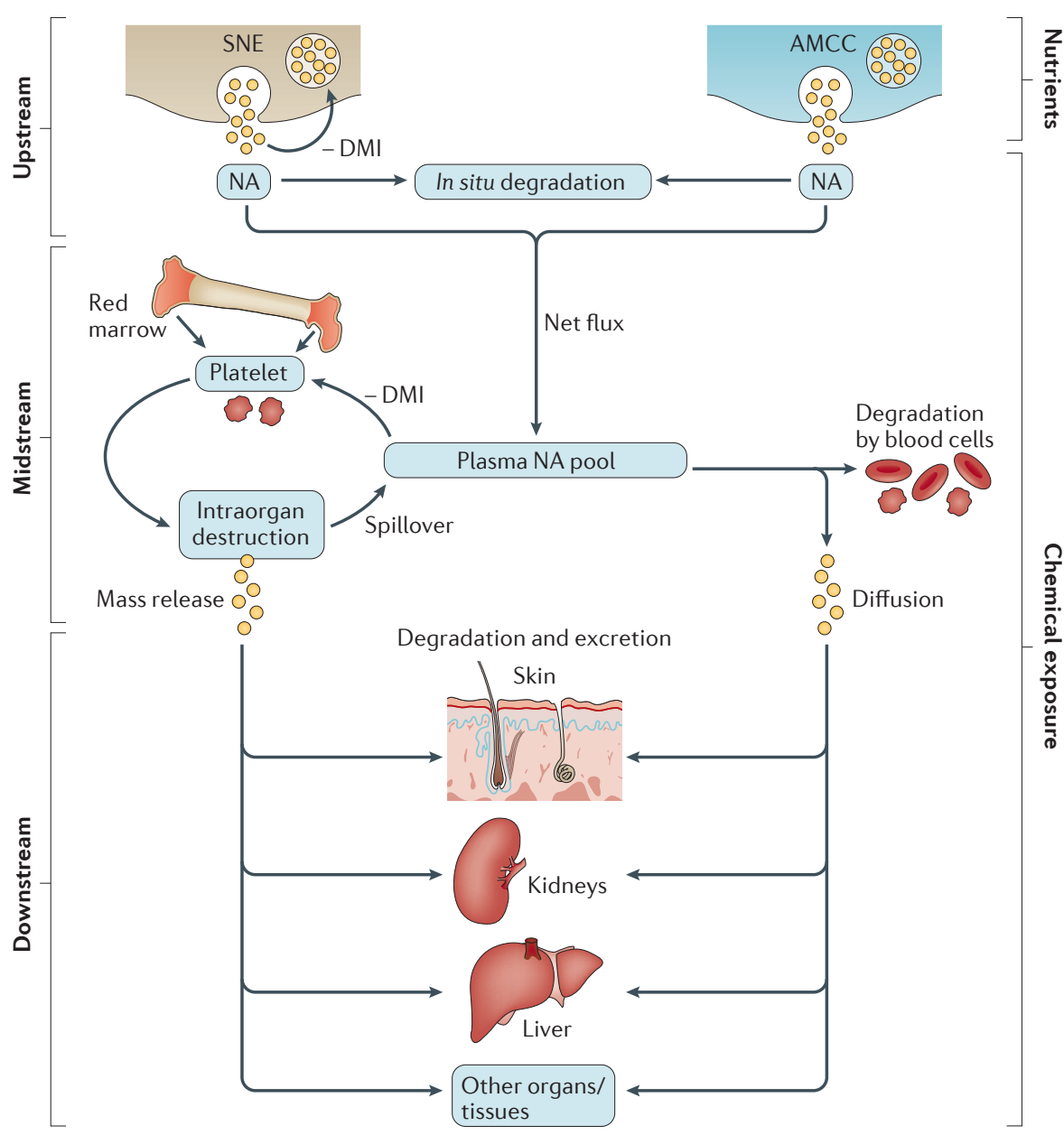

Figure 1 | Origin and fate of plasma noradrenaline (NA). Peripheral NA metabolism can be divided into three stages: postrelease metabolism in sympathetic nerve endings (SNE), adrenal medullary chromaffin cells (AMCC), and their target tissues (upstream); metabolism in the blood (midstream); and metabolism in detoxifying and excretory organs (downstream). Platelets take up and store very high concentrations of NA and thus serve as containers to transport NA to detoxifying/excretory organs, where it is released during platelet destruction. Nutrients enhance sympathetic activity by providing energy, neurotransmitter precursor amino acids, and neurotransmitter-synthesizing cofactors, while chemicals inhibit NA degradation and inactivation by competing for the body's limited detoxification resources (for example, methyl groups). DMI, desipramine. 


\section{CORRESPONDENCE}

platelets in lowering plasma noradrenaline and blood pressure. Unfortunately, the dynamic contribution of platelet noradrenalinedegrading enzymes was not taken into account in previous studies on the relationship between plasma and platelet noradrenaline levels, leading to inaccurate explanations on platelet neurotransmitter flux.

We disagree with the argument (which is based only on platelet pool size) that platelets do not contribute substantially to plasma noradrenaline clearance. Besides renal disease, cirrhosis is also well-documented to increase both mean platelet volume ${ }^{9}$ and noradrenaline spillover ${ }^{5}$. Given that renal disease increases platelet destruction (renal thrombotic microangiopathy) and that the liver is a major platelet-destroying organ ${ }^{6}$, a plausible explanation for these phenomena is that the noradrenaline concentrated in platelets cannot be effectively eliminated during intraorgan platelet destruction, consequently leading to increased noradrenaline spillover.
In conclusion, platelet overload might be a major cause of increased noradrenaline spillover in hypertension, reflecting detoxifying/ excretory insufficiency that cannot meet the needs of the sympathetic system ${ }^{10}$. The arguments by Barber-Chamoux and Esler ${ }^{1}$ were based on an incomplete understanding of platelet function.

Shi-Sheng Zhou is at the Institute of Basic Medical Sciences, Medical College, Dalian University, Dalian Economic and Technological Development Zone, Dalian 116622, China.

Yiming Zhou is at the Renal Division, Department of Medicine, Brigham and Women's Hospital, Harvard Institutes of Medicine, Harvard Medical School, 75 Francis Street, Boston, Massachusetts 02115, USA.

Correspondence to S.-S.Z. zhouss@ymail.com doi: 10.1038 /nrcardio. 2016.145 Published online 22 Sep 2016

. Barber-Chamoux, N. \& Esler, M. D. Platelet norepinephrine uptake is unrelated to renal denervation. Nat. Rev. Cardiol. http//dx.doi.org/ nrcardio.2016.144 (2016).
2. Zhou, S. S. \& Zhou, Y. Renal denervation for resistant hypertension: the wrong target? Nat. Rev. Cardiol. 13 388 (2016).

3. Gulati, R. et al. The rise, fall, and possible resurrection of renal denervation. Nat. Rev. Cardiol. 13, 238-244 (2016).

4. Zhou, S. S., Zhou, Y. M., Li, D. \& Chen, N. N. Preeclampsia and future cardiovascular risk: a point of view from the clearance of plasma vasoactive amines. Hypertens. Pregnancy 35, 1-14 (2016).

5. Esler, M. et al. Overflow of catecholamine neurotransmitters to the circulation: source, fate, and functions. Physiol. Rev. 70, 963-985 (1990).

6. Heyns, A. D. et al. Kinetics, distribution and sites of destruction of ${ }^{111}$ Indium-labelled human platelets. Br. J. Haematol. 44, 269-280 (1980).

7. Esler, M. D. et al. Effects of desipramine on sympathetic nerve firing and norepinephrine spillover to plasma in humans. Am. J. Physiol. 260 R817-R823 (1991).

8. Mielke, K. \& Strobel, G. The potential of intact human platelets for sulfoconjugation of norepinephrine in vitro. Life Sci. 55, 1657-1663 (1994).

9. Girleanu, I. et al. The risk of thrombotic events in patients with liver cirrhosis. Rev. Med. Chir. Soc. Med. Nat. Iasi 116, 991-996 (2012).

10. Zhou, S. S. \& Zhou, Y. Renal sympathetic hyperactivity in hypertension: true or false? Circ. Res. 119 e33-e34 (2016).

Competing interests statement

The authors declare no competing interests. 